\title{
Retraction Note to: Substrates specificity of tannase from Streptomyces sviceus and Lactobacillus plantarum
}

\author{
Dan Wang ${ }^{1+}$, Yao Liu' ${ }^{2+}$, Die Lv ${ }^{1 \dagger}$, Xueli Hu' , Qiumei Zhong ${ }^{1}$, Ye Zhao ${ }^{1 *}$ and Mingbo Wu ${ }^{1 *}$ (D)
}

\section{Retraction to: AMB Expr (2018) 8:147}

https://doi.org/10.1186/s13568-018-0677-1

The Editor-in-Chief has retracted this article (Wang et al. 2018) because the authors do not have ownership of the data they report. An investigation by the Commonwealth Scientific and Industrial Research Organisation (CSIRO) has concluded that the data reported in this article are the sole property of the CSIRO. Mingbo Wu agrees with this retraction. Dan Wang, Yao Liu, Die Lv, Xueli Hu, Qiumei Zhong and Ye Zhao have not responded to correspondence about this retraction.

\section{Publisher's Note}

Springer Nature remains neutral with regard to jurisdictional claims in published maps and institutional affiliations.

Received: 10 April 2019 Accepted: 10 April 2019

Published online: 19 April 2019

\section{Reference}

Wang D, Liu Y, Lv D, Hu X, Zhong Q, Zhao Y, Wu M (2018) Substrates specificity of tannase from Streptomyces sviceus and Lactobacillus plantarum. AMB Expr 8:147

The original article can be found online at https://doi.org/10.1186/s1356 8-018-0677-1.

\section{Author details}

${ }^{1}$ School of Bioscience and Biotechnology, Chengdu Medical College, 783

Xindu Avenue, Chengdu 610500, People's Republic of China. ${ }^{2}$ Laboratory of Veterinary, Drug Residue Prevention and Control Technology of Animal-derived Food, Chengdu Medical College, 783 Xindu Avenue, Chengdu 610500, People's Republic of China.

\footnotetext{
*Correspondence: zhaoye525@qq.com; wumingboscu@163.com †Dan Wang, Yao Liu and Die Lv contributed equally to this paper ${ }^{1}$ School of Bioscience and Biotechnology, Chengdu Medical College, 783 Xindu Avenue, Chengdu 610500, People's Republic of China

Full list of author information is available at the end of the article
} provided you give appropriate credit to the original author(s) and the source, provide a link to the Creative Commons license, and indicate if changes were made. 